\title{
Dynamics of the Shift Transformation on the GMLSm
}

\author{
Anandaram Burhagohain ${ }^{1}$ and Ranu Paul ${ }^{2}$ \\ ${ }^{1}$ Department of Mathematics, Gauhati University, Guwahati \\ ${ }^{2}$ Department of Mathematics, Pandu College, Guwahati Assam, India
}

\begin{abstract}
The topological Markov chain $\sigma_{A}$ on the Golden Mean Lookalike Shift of order $m$ [GMLSm] is a very typical topological discrete system possessing rich dynamical properties. In this paper we establish some results in connection with the chaotic properties of this Markov chain. In particular, we prove that it is Devaney chaotic (DevC), Auslander-Yorke chaotic and generically $\delta$-chaotic. Further, it has been shown that $\sigma_{A}$ has chaotic as well as modified weakly chaotic dependence on initial conditions. Moreover, the zeta function for this Markov chain has been derived.
\end{abstract}

Keywords: Shift Space, Shift Map, Devaney Chaos, Topological Transitivity, Topological Mixing, Topological Markov chain, Topological Conjugacy, Sensitivity Dependence.

\section{Introduction}

Symbolic representation of dynamical systems by means of Markov partitions [1, 2, 3] enable us to study the systems in a more comfortable way. General dynamical systems, which are in general too complicated to handle with, in this case are represented by sequence spaces, one-sided or two-sided, which are comparatively easier to analyse than the original ones. These sequence spaces, generally called shift spaces [1,4], give us some handy tools of mathematics. One can predict some complicated dynamical aspects of a system by simply studying its representative symbolic space. We feel more advantageous when the representing symbol space is a shift of finite type [1], also called topological Markov shift or simply a Markov shift [5]. For, these types of shifts are always represented by directed graphs which are equivalent to transition matrices and hence one can fruitfully employ the notions and results of graph theory as well as those of linear algebra. The relation between directed graphs and their transition matrices is like both way traffic. This is because of the fact that from a directed graph with $n$ vertices one can always obtain a definite transition matrix of order $n$ and conversely from a transition matrix of order $n$, one can have a definite graph with $n$ vertices up to graph isomorphism.

Fruitful symbolic representation of a system is not always possible for every dynamical system. It has been found that such representations are possible in the family of hyperbolic toral automorphisms [2] and in some other special systems like Axiom A systems [4]. The most expected situation is the case of getting a topological conjugacy $[1,2,6]$ between the map describing the original dynamical system and the associated topological Markov chain [2,7]. This is rarely encountered in real situations. But the arrival at this ideal situation is always considered as the landing in a very comfortable zone. Because, after arriving at this arena, one can say all about the dynamical behaviours of the original system. This is because of the fact that topologically conjugate systems are identical or the same at least in the topological sense. So, to study the general dynamical systems in the light of Markov chains representing them, one needs to study the dynamical aspects of various topological Markov chains beforehand. The Golden Mean Lookalike shifts (GMLS)[8] forms a very special class of topological Markov shifts. In [8], we have the definition of Golden Mean Lookalike Shifts (GMLS) and for this class Mangang calculated the topological entropy there. Here, in this paper, we have discussed some dynamical aspects of this topological Markov chain. Mainly, we have established that the shift transformation $\sigma_{A}$ on GMLSm $(m(\geq 2) \in \mathrm{N})$ is Devaney chaotic (DevC) [6, 9], Auslander-Yorke chaotic [10] and generically $\delta$-chaotic $[10,11]$. We have also established that $\sigma_{A}$ has chaotic and modified weakly chaotic dependence on initial conditions [10,11]. Further we have derived the zeta function [1] for this Markov chain.

\section{Basic Definitions, Discussions And Results:}

Definition 2.1: Li-Yorke Pairs [10, 12]: For a topological dynamical system $(X, T)$, a pair $(y, z) \in X^{2}$ is called a Li-Yorke pair with modulus $\delta>0$ if $\limsup _{n \rightarrow \infty} d\left(T^{n}(y), T^{n}(z)\right) \geq \delta$ and

$\liminf _{n \rightarrow \infty} d\left(T^{n}(y), T^{n}(z)\right)=0$. The set of all Li-Yorke pairs in $X$ is usually denoted by $L Y(T, \delta)$.

Definition 2.2: Weakly and modified weakly chaotic dependence on initial conditions: A dynamical system $(X, T)$ is called weakly (resp. modified weakly) chaotic dependence on initial conditions if for any $x \in X$ 
and every neighbourhood $N(x)$ of $x$, there are $y, z \in N(x)$ [ $y \neq x, z \neq x$ in modified weakly case] such that $(y, z) \in X^{2}$ is Li-Yorke.

Definition 2.3: Generically $\delta$-Chaotic maps: A continuous transformation $T: X \rightarrow X$ on a compact metric space $X$ is called generically $\delta$-chaotic if $L Y(T, \delta)$ is residual in $X^{2}$.

Definition 2.4: Essential Graphs [1]: A graph $G$ is essential if no vertex of it is stranded. i.e., there exists no vertex $v_{i}$ in $G$ such that either no edge start at $v_{i}$ or no edge terminate at $v_{i}$.

Definition 2.5: Irreducible and Aperiodic Matrices [1]: A transition matrix or a 0-1square matrix $A$ is said to be irreducible if for any $i, j \in \mathrm{N}, 1 \leq i, j \leq m, \exists n \in \mathrm{N}$ (possibly dependent on $i, j \in \mathrm{N}$ ) such that $\left(A^{n}\right)_{i j}>0$. i.e., the $(i, j)^{\text {th }}$ entry $\left(A^{n}\right)_{i j}$ of the matrix $A^{n}$ is positive.

On the other hand, a transition matrix is aperiodic (transitive) if there exists an $n \in \mathrm{N}$ such that for any $1 \leq i, j \leq m,\left(A^{n}\right)_{i j}>0$. i.e. the matrix $A^{n}$ is positive. From the definitions it immediately follows that $a n$ aperiodic matrix is always irreducible.

\section{1: The bi-sided full m-shift $\Sigma_{m}$, Shift spaces, Shifts of finite type and Sub-shifts:}

For $m(\geq 2) \in N$, the bi-sided full $\boldsymbol{m}$-shift $[1,13]$ over the alphabet $A=\{0,1,2, \ldots, m-1\}$ is the set $\left\{\left(a_{i}\right)_{i=-\infty}^{\infty}: a_{i} \in A=\{0,1,2, \ldots . ., m-1\}, i \in Z\right\}$ of all the bi-infinite sequences of $m$-symbols, also called the letters. This shift is shortly denoted by $\mathrm{X}_{[m]}$ or $\sum_{m}$ or $A^{Z}$. In expanded form the point $x=\left(x_{i}\right)_{i=-\infty}^{\infty}$ is generally denoted as $x=\ldots \ldots x_{-3} x_{-2} x_{-1} \cdot x_{0} x_{1} x_{2} x_{3} \ldots .$. where $x_{i}^{\prime s} \in A$.

A finite sequence $x_{i} x_{i+1} \ldots x_{j}$ of letters from the alphabet $A$, denoted by $x_{[i, j]}$, is called a block or a word of length $(j-i+1)$ and for $n \in \mathrm{N}$, the block $x_{[-n, n]}=x_{-n} \ldots x_{0} \ldots x_{n}$ is called the central $(2 n+1)$-block of the point $x=\left(x_{i}\right)_{i=-\infty}^{\infty} \in \Sigma_{m}$. Central blocks of points in a shift are more important in the study of symbolic dynamics.

A shift space (or simply a shift) $X$ is a subset of a full shift $\Sigma_{m}=A^{Z}$ such that $X=X_{F}$ where $F$ is some collection of blocks, called forbidden blocks, over the alphabet $A$ each of which does not occur in any sequence in $X=X_{F}$. If $F$ is finite, then $X=X_{F}$ is called a shift of finite type or a topological Markov shift [1]. For two shift spaces $X$ and $Y$, if $Y \subseteq X$ then $Y$ is known as a sub-shift of $X$. If $B_{n}(X)$ denotes the collection of all the allowed (not forbidden)n-blocks occurring in the points in $X$, then the $\operatorname{set} B(X)=\bigcup_{n=1}^{\infty} B_{n}(X)$ is called the language of $X . X$ is an irreducible shift if for every pair of blocks $u, v \in B(X)$ there is a block $w \in B(X)$ such that $u w v \in B(X)$.

\section{2: Graphs and their Adjacency Matrices, Edge Shifts and Vertex Shifts:}

The well-known relations (i) $A=\mathrm{A}\left(\mathrm{G}_{A}\right)$ and (ii) $G \cong \mathrm{G}\left(\mathrm{A}_{G}\right)$, where $A$ is the adjacency matrix of the graph $G$, allow one to use freely a graph $G$ or its adjacency matrix $A$ for the specification of the underlying graph, whichever seems more convenient in the context.If $E$ is the edge set and $A$ is the adjacency matrix for a graph $G$, then the $\boldsymbol{e}$ edge shift [1] corresponding to $G$, denoted by $\mathrm{X}_{G}$ or $\mathbf{X}_{\boldsymbol{A}}$, is the shift space over the alphabet $E$ such that

$$
\mathrm{X}_{A}=\mathrm{X}_{G}=\left\{e=\left(e_{i}\right)_{i \in Z}: t\left(e_{i}\right)=i\left(e_{i+1}\right), e^{\prime s} \in E\right\},
$$

Where $t\left(e_{i}\right)$ and $i\left(e_{i+1}\right)$ respectively denote the terminal vertex of the edge $e_{i}$ and the initial vertex of the edge $e_{i+1}$. From the above definition one can clearly understand the strong connection between graphsand shifts.Golden Mean Shift stands as an example that every shift of finite type is not always an edge shift. But by using higher block presentation, any shift of finite type can be recoded to have an edge shift.An alternative description of a shift of finite type can be given by using transition matrices.If $B$ be a transition matrix (0-1 matrix) of order $m \times m$, then it is the adjacency matrix of a graph $G$ containing at most one edge between any two vertices. The shift space denoted by $\hat{X}_{B}=\hat{X}_{G}$ is called the vertex shift defined as follows: 


$$
X_{B}=\hat{X}_{G}=\left\{x=\left(x_{i}\right)_{i \in Z} \in A^{Z}: B_{x_{i} x_{i+1}}=1, \forall i \in Z, A=\{1,2,3,4, \ldots, m\}\right\}
$$

These shifts are 1-step shifts of finite type. The vertex shift $\hat{X}_{B}$ is not other than the shift space $X_{F}$ where $F=\left\{i j: B_{i j}=0, i, j \in Z\right\}$. The vertex shift corresponding to the transition matrix $B$ is also denoted by $\Sigma_{B}$. The following propositions have been extensively used in proving the results in next sections.

Proposition.2.1 [8]: A topological dynamical system $T: X \rightarrow X$ is topologically transitive if for every pair of non-empty open sets $U$ and $V$ of $X$, there exists a positive integer $n \in \mathrm{N}$ such that $T^{n}(U) \cap V \neq \phi$.

Proposition 2.2[7]: Let $X$ be a compact metric space and $T: X \rightarrow X$ be a continuous topologically mixing map. Then, $T$ is also topologically weak mixing.

Proposition 2.3[13]: Let $T: X \rightarrow X$ be a continuous map on a compact metric space $X$. If $T$ is topologically weak mixing, then it is generically $\delta$-chaotic on $X$ with $\delta=\operatorname{diam}(X)$.

Proposition: 2.4[1]: If $G$ is a graph with adjacency matrix $A$, then the associated edge shift $X_{G}=X_{A}$ is a 1-step shift of finite type.

Proposition: 2.5[1]: If $G$ is a graph, then there is a unique sub-graph $H$ of $G$ such that $H$ is essential and $X_{G}=$ $X_{H}$.

Proposition: 2.6[1]: Let $G$ be a graph with adjacency matrix $A$ and $m(\geq 0) \in N$. Then,

(i) The number of paths of length $m$ from I to $J$ is $\left[A^{m}\right]_{I J}$, the $(I, J)^{\text {th }}$ entry of $A^{m}$.

(ii) The number of cycles of length $m$ in $G$ is $\operatorname{tr}\left(A^{m}\right)$, the trace of $A^{m}$ and this equals the number of points in $X_{G}$ with period $m$.

2.3: The Golden Mean shift, Golden Mean Lookalike Shifts and cylinder sets:

The Golden Mean shift [1] is a Markov shift (shift of finite type) $X_{F}$ which is a sub-shift of the full 2-shift $\mathrm{X}_{[2]}=\Sigma_{2}=\left\{x=\left(x_{i}\right)_{i=-\infty}^{\infty}: x_{i} \in\{0,1\}\right\}$ with the forbidden class $F$ given by $F=\{11\}$. More precisely, the Golden Mean shift contains all the bi-infinite binary sequences which do not contain the 2-block 11. This shift is described by the transition matrix $A$ and Graph $G$ :

$$
A=\left[\begin{array}{ll}
1 & 1 \\
1 & 0
\end{array}\right]
$$

\section{Adjacency matrix of Golden Mean shift}

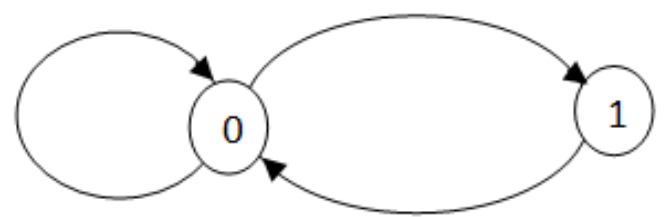

Fig.1 Graph of GMLS4 with alphabet $A=\{0,1,2,3\}$

The eigen values of the above transition matrix $A$ describing the Golden Mean shift are $\lambda_{1}=\frac{1+\sqrt{5}}{2}$ and $\lambda_{2}=\frac{1-\sqrt{5}}{2}$. For geometric reasons, the number $\lambda_{1}=\frac{1+\sqrt{5}}{2}$ is known as the golden mean or golden ratio and that is the reason why $X_{F}$ is called the Golden Mean shift. This shift is also known as the Fibonacci shift. For, the number of allowed or admissible $n$-blocks, $n \in \mathrm{N}$, are the numbers starting from the third term of the Fibonacci sequence, namely, there are two 1-blocks, three 2-blocks, five 3-blocks, eight 4-blocks,........etc. We notice that all the entries in the first row and first column of the transition matrix describing the Golden Mean shift are 1's and the other entries are 0's. Likewise we can think of a transition matrix of order $m(>2) \in \mathrm{N}$ such that every entry in the first row and first column is 1 and all the remaining entries are 0 's. The shift described by this transition matrix is defined as the Golden Mean Lookalike shift of order $m$ and shortly it is denoted by GMLS $m$. That is, a Golden Mean Lookalike Shift of order $m$ is a shift $X_{F}$ of finite type over the alphabet $A=\{0,1,2, \ldots, m-1\}$ where $F=\{i j: i(>0), j(>0) \in A\}$. Since, each block in the forbidden class $F$ is of length 2, so GMLS $m$ is a 1-step Markov shift. For $m=3,4,5, \ldots$, the respective Golden Mean Lookalike shifts are GMLS3, GMLS4, GMLS5, GMLS6,..., etc. The adjacency matrix $A$ and the representing graph $G$ of the GMLS4 are shown below: 

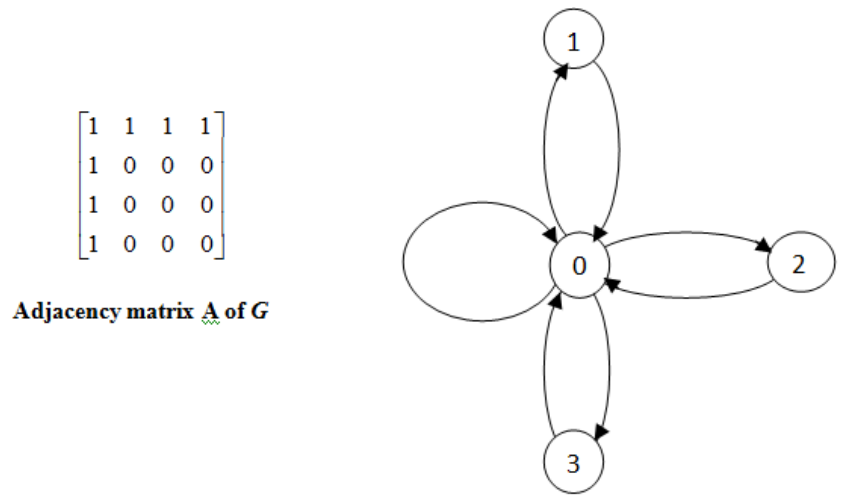

Fig.2: Graph $G$ of GMLS4 with alphabet $A=\{0,1,2,3\}$

Clearly GMLS4 is the vertex shift of the transition matrix $A$ of order 4 such that $A=\left(A_{i j}\right)_{4 \times 4}$ where $A_{i j}=1$ for $i=1$ or $j=1$ and $A_{i j}=0$ for $i, j>1$

Similarly, GMLS $m$ is the vertex shift of the transition matrix $A$ of order $m$ given by $A=\left(A_{i j}\right)_{m \times m}$ where $A_{i j}=1$ for $i=1$ or $j=1$ and $A_{i j}=0$ for $i, j>1$

One fact to be noted particularly is that in GMLSm, 0 can precede and follow every other letter of the alphabet $A=\{0,1,2, \ldots, m-1\}$, but no non-zero letter can precede and follow every other non-zero letter of the alphabet.

Now, for $\rho>1$ and $x=\left(x_{i}\right)_{i=-\infty}^{\infty}, y=\left(y_{i}\right)_{i=-\infty}^{\infty} \in \Sigma_{A}$, the mapping $d_{\rho}: \Sigma_{A} \times \Sigma_{A} \rightarrow R$ defined by

$$
d_{\rho}(x, y)=\left\{\begin{array}{l}
\rho^{-k} \text { if } x \neq y \text { and } k \in N \text { is greatest s.t. } x_{[-k, k]}=y_{[-k, k]} \\
1 \quad \text { if } x_{0} \neq y_{0} \\
0 \quad \text { if } x=y
\end{array}\right.
$$

is a metric for $\Sigma_{A} . \Sigma_{A}$ is a compact metric space[13] under this metric [1]. Again, the shift map $\sigma_{A}$ on $\Sigma_{A}$ defined by $\sigma_{A}(x)=\ldots \ldots . x_{-2} x_{-1} x_{0} \cdot x_{1} x_{2} x_{3} \ldots \ldots .$. is a continuous map [1,6].Hence $\left(\Sigma_{A}, \sigma_{A}\right)$ is a topological dynamical system (TDS)[13].

From the definition of the metric $d_{\rho}: \Sigma_{A} \times \Sigma_{A} \rightarrow R$, it follows that points in the shift space $\Sigma_{A}$ are close to each other if they agree in their large central blocks.

Now, we formally define the terms cylinders, admissible cylinders, symmetric cylinders and admissible symmetric cylinders which are very essential in studying shift dynamical systems.

For $r, s \in \mathrm{N}$ and $a_{i} \in\{0,1,2, \ldots \ldots, m-1\}$ with $-r \leq i \leq s$, a cylinder $C_{-r, s}\left(a_{-r}, a_{-r+1}, \ldots \ldots . ., a_{s}\right)$ is a subset of $\Sigma_{m}$ defined as:

$$
C_{-r, s}\left(a_{-r}, a_{-r+1}, \ldots \ldots . ., a_{s}\right)=\left\{x=\left(x_{i}\right)_{i=-\infty}^{\infty} \in \Sigma_{m}: x_{i}=a_{i}, \forall-r \leq i \leq s\right\}
$$

For $r \in \mathrm{N}$, the cylinder $C_{-r, r}\left(a_{-r}, a_{-r+1}, \ldots \ldots ., a_{r}\right)$ is called a symmetric cylinder. In case of a Markov shift $\Sigma_{A} \subset \Sigma_{m}$ corresponding to a transition matrix $A$, the cylinders $C_{-r, s}\left(a_{-r}, \ldots ., a_{s}\right)$ and

$C_{-r, r}\left(a_{-r}, a_{-r+1}, \ldots ., a_{r}\right)$ are respectively called an admissible cylinder and an admissible symmetric cylinder if $A_{a_{i} a_{i+1}}=1, \forall-r \leq i<s$.

The following well known propositions on cylinder sets are very important in application point of view.

Proposition: 2.7: If $\rho>2 m-1$ and $n \in \mathrm{N}$, then for $\varepsilon=1 / \rho^{n}, C_{-n, n}\left(x_{-n}, \ldots \ldots, x_{n}\right)=B_{d_{\rho}}\left(x, 1 / \rho^{n}\right)$ where $x=\left(x_{i}\right)_{i=-\infty}^{i=\infty} \in \Sigma_{m}$ contains the central block $x_{[-n, n]}=x_{-n} \ldots . . x_{-1} \cdot x_{0} \ldots \ldots . x_{n}$.

Proposition: 2.8: If $\rho>2 m-1$, then any non-empty open set $U \subset \Sigma_{m}$ contains a symmetric cylinder $C_{-n, n}\left(a_{-n}, \ldots ., a_{n}\right)$. 
Proposition: 2.9: If $\rho>2 m-1$, then any non-empty open set $U \subset \Sigma_{A}$ contains an admissible symmetric cylinder $C_{-n, n}\left(a_{-n}, \ldots . ., a_{n}\right)$.

\section{The Main Results}

Proposition: 3.1[2]: If $\sigma_{A}: \Sigma_{A} \rightarrow \Sigma_{A}$ be a topological Markov chain corresponding to the transition matrixA, then,

(i) A is irreducible if and only if $\sigma_{A}: \Sigma_{A} \rightarrow \Sigma_{A}$ is topologically transitive.

(ii) If $A$ is aperiodic (transitive), then, $\sigma_{A}: \Sigma_{A} \rightarrow \Sigma_{A}$ is topologically mixing.

Proof: In the proof of this proposition we shall use the following Lemma.

Lemma [2]: If $A^{n}>0$ for some $n \in \mathrm{N}$, then for any integer $r>n$ we also have that $A^{r}>0$. Proof of the Lemma: Here we mainly use the concepts of graph of the transition matrix $A$ to prove this Lemma.

Let $m$ be the order of the matrix $A$ and $A^{n}>0$ for some $n \in \mathrm{N}$. This means that for every $j \in \mathrm{N}, 1 \leq j \leq m$, there exists $k_{j} \in \mathrm{N}, 1 \leq k_{j} \leq m$ such that $A_{k_{j} j}=1$. If not, then $A_{k j}=0$ for all $k \in \mathrm{N}$ with $1 \leq k \leq m$, and in that case the vertex $v_{j}$ of the corresponding graph of $A$ cannot be reached from any other vertex $v_{k}$. Consequently, we cannot have any path of length $n$ reaching the vertex $v_{j}$. This gives that $A_{k j}^{n}=0$ which contradicts our assumption that $A_{i j}^{n}>0\left(\because A^{n}>0\right)$. Now, by induction we establish that for any $r(\geq n) \in \mathrm{N}$, $A^{r}>0$. The result is already true for $r=n$ by our hypothesis. Suppose, it is true for $r(>n) \in \mathrm{N}$ such that $A^{r}>0$ and also assume that $1 \leq i, j \leq m$. Then, by our previous remark, for every $1 \leq j \leq m$, there exists $k_{j} \in \mathrm{N}$ such that $A_{k_{j} j}=1$. Again, for every other $1 \leq k \leq m$, we have $A_{k j} \geq 0$. So, clearly we have that

$$
A_{i j}^{r+1}=\sum_{r=1}^{m} A_{i k}^{r} A_{k j} \geq A_{i k_{j}}^{r} A_{k_{j} j}=A_{i k_{j}}^{r} \cdot 1=A_{i k_{j}}^{r}>0\left[\because A^{r}>0 \Rightarrow A_{i k_{j}}^{r}>0\right] .
$$

Therefore, $A^{r+1}>0$ when $A^{r}>0$ and hence by induction the Lemma follows.

\section{Proof of the proposition:}

Part (i): Let $A$ be irreducible. We now show that $\sigma_{A}: \Sigma_{A} \rightarrow \Sigma_{A}$ is topologically transitive. For this we show that for any non-empty open sets $U, V \subseteq \Sigma_{A}, \exists M \in \mathrm{N}$ such that $\sigma_{A}^{M}(U) \cap V \neq \phi$.

We first fix $\rho>2 m-1$. By proposition 2.6, for $U, V \subseteq \Sigma_{A}$ there exist admissible symmetric cylinders $C_{-r, r}\left(x_{-r}, \ldots, x_{r}\right) \subseteq U$ and $C_{-s, s}\left(y_{-s}, \ldots, y_{s}\right) \subseteq V$.

Now using the blocks $x_{[-r, r]}=x_{-r} \ldots x_{r}$ and $y_{[-s, s]}=y_{-s} \ldots . y_{s}$, we construct a point $z \in \Sigma_{A}$. Take $i=x_{r}$ and $j=y_{-s}$. By irreducibility of $A$, for these $i$ and $j$, there exists $n \in \mathrm{N}$ such that $A_{i j}^{n}>0$. That is, there is a path of length $n$ in $\mathrm{G}_{A}$ that connects $v_{x_{r}}$ to $v_{y_{-s}}$. Let the digits describing this path be $x_{r}=z_{0}, z_{1}, \ldots ., z_{n-1}, z_{n}=y_{-s}$. Evidently, for each $i, 0 \leq i \leq n-1$, we have $A_{z_{i} z_{i+1}}=1$. Now, consider the point $z \in \Sigma_{A}$ such that

$$
z=\ldots \ldots x_{-r} \ldots \ldots x_{-2} x_{-1} \cdot x_{0} x_{1} \ldots \ldots x_{r} z_{1} z_{2} \ldots \ldots z_{n-1} y_{-s} \ldots \ldots . y_{s} \ldots \ldots .
$$

Since $z$ contains the central block $x_{-r} \ldots x_{r}$, so $z \in C_{-r, r}\left(x_{-r}, \ldots, x_{r}\right) \subseteq U$. Further, if we take $M=r+n+s$ , then $\sigma_{A}^{M}(z)=\ldots \ldots y_{-s} \ldots . . y_{-1} \cdot \underbrace{y_{0} \ldots . . . y_{s} \ldots \ldots .}_{i=0}$ and so $\sigma_{A}^{M}(z) \in C_{-s, s}\left(y_{-s}, \ldots . ., y_{s}\right) \subseteq V$. So, it follows that $z \in U \cap \sigma_{A}^{-M}(V) \Leftrightarrow \sigma_{A}^{M}(U) \cap V \neq \phi$. Hence, $\sigma_{A}: \Sigma_{A} \rightarrow \Sigma_{A}$ is topologically transitive.

Conversely, assume that $\sigma_{A}: \Sigma_{A} \rightarrow \Sigma_{A}$ be topologically transitive. We show $A$ is irreducible. Let $1 \leq i, j \leq m$ and consider the cylinders $C_{0}(i)=\left\{x \in \Sigma_{A}: x_{0}=i\right\}$ and $C_{0}(j)=\left\{y \in \Sigma_{A}: y_{0}=j\right\}$. Since, cylinder sets are always open, so, we take $U=C_{0}(i)$ and $V=C_{0}(j)$ as open sets. Then by transitivity of the $\operatorname{map} \sigma_{A}: \Sigma_{A} \rightarrow \Sigma_{A}$, there exists $n \in \mathrm{N}$ such that $\sigma_{A}^{n}(U) \bigcap V \neq \phi$. 


$$
\text { Now, } \begin{aligned}
\sigma_{A}^{n}(U) \cap V \neq \phi & \Leftrightarrow U \cap \sigma_{A}^{-n}(V) \neq \phi \\
& \Leftrightarrow \exists z \in U \cap \sigma_{A}^{-n}(V) \\
& \Leftrightarrow \exists z \text { such that } z \in U=C_{0}(i) \text { and } z \in \sigma_{A}^{-n}\left(V=C_{0}(j)\right) \\
& \Leftrightarrow \exists z \text { such that } z_{0}=i \text { and } z_{n}=j
\end{aligned}
$$

Thus $z \in U \subseteq \Sigma_{A}$ is an element that describes a bi-infinite path on the graph $\mathrm{G}_{A}$ of the transition matrix $A$ such that $z_{0}=i, z_{n}=j$.This gives a path of length $n$ connecting the vertex $v_{i}$ to the vertex $v_{j}$. So, for all $i, j$ with $1 \leq i, j \leq m$, there exists $n \in \mathrm{N}$ such that $A_{i j}^{n}>0$. Therefore, $A$ is irreducible.

Part (ii): Let $A$ be aperiodic. Then, by definition, $\exists n \in \mathrm{N}$ such that $A^{n}>0$. We prove that the map $\sigma_{A}: \Sigma_{A} \rightarrow \Sigma_{A}$ is topologically mixing. For this we need to prove that for any pair of non-empty open sets $U, V \subseteq \Sigma_{A}, \exists M_{0} \in \mathrm{N}$ such that $\sigma_{A}^{M}(U) \cap V \neq \phi$ for all $M \geq M_{0}$.

Both $U, V \subseteq \Sigma_{A}$ being non-empty open sets, these open sets must contain admissible symmetric cylinders $C_{-k, k}\left(x_{-k}, \ldots ., x_{k}\right) \subseteq U$ and $C_{-l, l}\left(y_{-l}, \ldots, y_{l}\right) \subseteq V$. Let $M_{0}=n+k+l$. If we take $M \in \mathrm{N}$ such that $M \geq M_{0}$, then, $M=m+k+l$, where $m \geq n$.

Also since $m \geq n$ and $A^{n}>0$, so, by the above Lemma we have that $A^{m}>0$. Then, $A_{x_{k} y_{-l}}^{m}>0$. Therefore, there exists a path of length $m$ from the vertex $x_{k}$ to the vertex $y_{-l}$. So, as in part (i), we can construct a point $z$ of the form $z=\ldots \ldots x_{-k} \ldots . . x_{-2} x_{-1} \cdot x_{0} x_{1} \ldots \ldots x_{k} z_{1} z_{2} \ldots \ldots z_{n-1} y_{-1}$ in $\Sigma_{A}$ such that $z \in U \cap \sigma_{A}^{-M}(V)$ and from this it immediately follows that $\sigma_{A}^{M}(U) \cap V \neq \phi$. This is true for any $M \geq M_{0}$. So, we conclude that $\sigma_{A}: \Sigma_{A} \rightarrow \Sigma_{A}$ is topologically mixing.

Theorem: 3.2: The shift map $\sigma_{A}: \Sigma_{A} \rightarrow \Sigma_{A}$ is topologically transitive as well as mixing.

Proof : ( i) $\sigma_{A}: \Sigma_{A} \rightarrow \Sigma_{A}$ is topologically transitive:

Let us first fix $\rho>2 m-1$.Then, consider any two non-empty open sets $U$ and $V$ in $\Sigma_{A}$. For these two nonempty open sets $U$ and $V$ in $\Sigma_{A}$, we show that there exists a positive integer $n$ such that $\sigma_{A}^{n}(U) \cap V \neq \phi . U$ and $V$ being non-empty, there exists two points $x$ and $y$ in $\Sigma_{A}$ such that $x=\left(x_{i}\right)_{i=-\infty}^{\infty} \in U$ and $y=\left(y_{i}\right)_{i=-\infty}^{\infty} \in V$. Again, since $U$ and $V$ are open sets in $\Sigma_{A}$, so there exists two open balls $B_{d_{\rho}}\left(x, r_{1}\right)$ and $B_{d_{\rho}}\left(y, r_{2}\right)$ such that $B_{d_{\rho}}\left(x, r_{1}\right) \subseteq U$ and $B_{d_{\rho}}\left(y, r_{2}\right) \subseteq V$. Then for the radii $r_{1}, r_{2}>0$, we can choose $n \in \mathrm{N}$ such that $\rho^{-n} \leq \min \left\{r_{1}, r_{2}\right\}$ and in this case we clearly have that $B_{d_{\rho}}\left(x, \rho^{-n}\right) \subseteq U$ and $B_{d_{\rho}}\left(y, \rho^{-n}\right) \subseteq V$. Also, since $\rho>2 m-1$, so $B_{d_{\rho}}\left(x, \rho^{-n}\right)=C_{-n, n}\left(x_{-n}, \ldots ., x_{n}\right)$ and $B_{d_{\rho}}\left(y, \rho^{-n}\right)=C_{-n, n}\left(y_{-n}, \ldots ., y_{n}\right)$. That is, the open balls $B_{d_{\rho}}\left(x, \rho^{-n}\right)$ and $B_{d_{\rho}}\left(y, r_{2}\right)$ thus obtained for the points $x=\left(x_{i}\right)_{i=-\infty}^{\infty} \in U$ and $y=\left(y_{i}\right)_{i=-\infty}^{\infty} \in V$ are nothing but are the admissible symmetric cylinders $C_{-n, n}\left(x_{-n}, \ldots ., x_{n}\right)$ and $C_{-n, n}\left(y_{-n}, \ldots ., y_{n}\right)$ respectively. Therefore, all the points in $B_{d_{\rho}}\left(x, \rho^{-n}\right)$ must agree with $x$ in the $(2 n+1)$ central block and all those of $B_{d_{\rho}}\left(y, \rho^{-n}\right)$ must agree with $y$ in the $(2 n+1)$-central block.

Now consider a point $z=\left(z_{i}\right)_{i=-\infty}^{\infty} \in \Sigma_{m}$ such that $z_{i}=x_{i}, \forall i \leq n, z_{n+1}=0$ and $z_{n+i}=y_{i-2-n}, \forall i \geq 2$. That is, $z=\left(z_{i}\right)_{i=-\infty}^{\infty}=x_{[-\infty,-1]} \cdot x_{[0, n]} 0 y_{[-n, \infty]} \in \Sigma_{A}$. Then $z$ agrees with $x$ in the $(2 n+1)$-central block and hence $z=\left(z_{i}\right)_{i=-\infty}^{\infty} \in C_{-n, n}\left(x_{-n}, \ldots, x_{n}\right)=B_{d_{\rho}}\left(x, \rho^{-n}\right)$. Again, clearly $\sigma_{A}^{2 n+2}(z)$ agrees with $y$ in the $(2 n+1)-$ central block and so $\sigma_{A}^{2 n+2}(z) \in B_{d_{\rho}}\left(y, \rho^{-n}\right)$. Also, since in a GMLS, 0 can precede and follow any letter of the alphabet and $x_{[-\infty, n]}, y_{[-n, \infty]} \in B\left(\Sigma_{A}\right)$, the language of $\Sigma_{A}$, so it follows that $z=\left(z_{i}\right)_{i=-\infty}^{\infty}=x_{[-\infty,-1]} \cdot x_{[0, n]} 0 y_{[-n, \infty]} \in \Sigma_{A}$. 
Thus $z \in B_{d_{\rho}}\left(x, \rho^{-n}\right) \subseteq U, \sigma_{A}^{2 n+2}(z) \in B_{d_{\rho}}\left(y, \rho^{-n}\right) \subseteq V$

$$
\begin{aligned}
& \Rightarrow \sigma_{A}^{2 n+2}(z) \in \sigma_{A}^{2 n+2}(U), \sigma_{A}^{2 n+2}(z) \in V \\
& \Rightarrow \sigma_{A}^{2 n+2}(z) \in \sigma_{A}^{2 n+2}(U) \cap V \\
& \Rightarrow \sigma_{A}^{2 n+2}(U) \cap V \neq \phi
\end{aligned}
$$

Thus for any two non-empty open sets $U$ and $V$ in $\Sigma_{A}$, there exists $M=2 n+2 \in \mathrm{N}$ such that $\sigma_{A}^{M}(U) \cap V \neq \phi$. Hence the self-map $\sigma_{A}: \Sigma_{A} \rightarrow \Sigma_{A}$ is topologically transitive.

(ii) $\sigma_{A}: \Sigma_{A} \rightarrow \Sigma_{A}$ is topologically mixing:

Let $\rho>2 m-1$ be fixed and $U, V$ be any two non-empty open sets in $\Sigma_{A}$. For topological mixing, here we need to prove that for the non-empty open sets $U$ and $V$, there exists $n_{0} \in \mathrm{N}$ such that

$\sigma_{A}^{k}(U) \cap V \neq \phi, \forall k(\in \mathrm{N}) \geq n_{0} . U$ and $V$ being non-empty, we have, $x=\left(x_{i}\right)_{i=-\infty}^{\infty} \in U$ and

$y=\left(y_{i}\right)_{i=-\infty}^{\infty} \in V$. Again, since $U$ and $V$ are open sets in $\Sigma_{A}$, there exists two open balls $B_{d_{\rho}}\left(x, r_{1}\right)$ and $B_{d_{\rho}}\left(y, r_{2}\right)$ such that $B_{d_{\rho}}\left(x, r_{1}\right) \subseteq U$ and $B_{d_{\rho}}\left(y, r_{2}\right) \subseteq V$. Now for the two radii $r_{1}, r_{2}>0$, we can choose $n \in \mathrm{N}$ such that $\rho^{-n} \leq \min \left\{r_{1}, r_{2}\right\}$. Then clearly we have that $B_{d_{\rho}}\left(x, \rho^{-n}\right) \subseteq U$ and $B_{d_{\rho}}\left(y, \rho^{-n}\right) \subseteq V$. Also, since $\rho>2 m-1$ has been fixed, so, we have that $B_{d_{\rho}}\left(x, \rho^{-n}\right)=C_{-n, n}\left(x_{-n}, \ldots, x_{n}\right)$ and $B_{d_{\rho}}\left(y, \rho^{-n}\right)=C_{-n, n}\left(y_{-n}, \ldots ., y_{n}\right)$. That is, the open balls $B_{d_{\rho}}\left(x, \rho^{-n}\right)$ and $B_{d_{\rho}}\left(y, \rho^{-n}\right)$ thus obtained for the points $x=\left(x_{i}\right)_{i=-\infty}^{\infty} \in U$ and $y=\left(y_{i}\right)_{i=-\infty}^{\infty} \in V$ are nothing but the admissible symmetric cylinders $C_{-n, n}\left(x_{-n}, \ldots ., x_{n}\right)$ and $C_{-n, n}\left(y_{-n}, \ldots ., y_{n}\right)$ respectively. Therefore, every point in $B_{d_{\rho}}\left(x, \rho^{-n}\right)$ must agree with $x$ in the $(2 n+1)$-central block and every point in $B_{d_{\rho}}\left(y, \rho^{-n}\right)$ must agree with $y$ in the $(2 n+1)$-central block. We now construct a sequence $\left\{z_{i}\right\}$ of points in $\Sigma_{A}$ with the help of $x, y$ and $n$ as follows:

$$
\begin{aligned}
& z_{1}=\ldots \ldots x_{-n} \ldots \ldots x_{-1} \cdot x_{0} \ldots \ldots x_{n} y_{-n} \ldots \ldots \ldots y_{n} y_{n+1} \ldots \ldots \ldots \\
& z_{2}=\ldots \ldots x_{-n} \ldots \ldots x_{-1} \cdot x_{0} \ldots \ldots x_{n} 0 y_{-n} \ldots \ldots y_{n} y_{n+1} \ldots \ldots \ldots \\
& z_{3}=\ldots \ldots x_{-n} \ldots \ldots x_{-1} \cdot x_{0} \ldots \ldots x_{n}(0)^{2} y_{-n} \ldots \ldots y_{n} y_{n+1} \ldots \ldots . \\
& z_{i}=\ldots \ldots x_{-n} \ldots . . x_{-1} \cdot x_{0} \ldots \ldots x_{n}(0)^{i-1} y_{-n} \ldots \ldots y_{n} y_{n+1} \ldots \ldots, i \geq 2,(0)^{i-1}=\underbrace{0000 \ldots 0}_{(i-1) \text { nos. }}
\end{aligned}
$$

Here, for every $i \geq 2, z_{i}$ is constructed by concatenating the words $x_{[-\infty, n]},(0)^{i-1}$ and $y_{[-n, \infty]}$. Again, for $i \geq 1$, since every $z_{i}$ agrees with $x$ at least in the $(2 n+1)$-central block, so we have that $z_{i} \in C_{-n, n}\left(x_{-n}, \ldots ., x_{n}\right)=B_{d_{\rho}}\left(x, \rho^{-n}\right) \subseteq U$. Also since 0 can precede and follow any letter of the alphabet in a GMLS and $x_{[-\infty, n]}, y_{[-n, \infty]} \in B\left(\Sigma_{A}\right)$, so, it follows that

$$
z_{i}=\ldots \ldots x_{-n} \ldots . . x_{-1} \cdot x_{0} \ldots . . x_{n}(0)^{i-1} y_{-n} \ldots . . y_{n} y_{n+1} \ldots \ldots=x_{[-\infty,-1]} \cdot x_{[0, n]}(0)^{i-1} y_{[-n, \infty]} \in \Sigma_{A}
$$

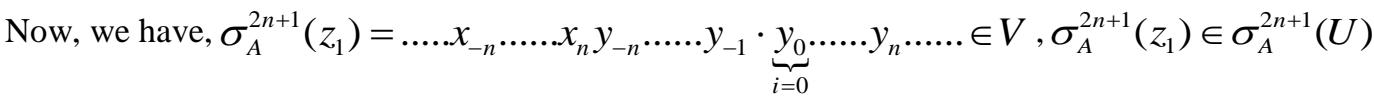

$$
\begin{aligned}
& \Rightarrow \sigma_{A}^{2 n+1}\left(z_{1}\right) \in \sigma_{A}^{2 n+1}(U) \cap V \\
& \Rightarrow \sigma_{A}^{2 n+1}(U) \cap V \neq \phi .
\end{aligned}
$$

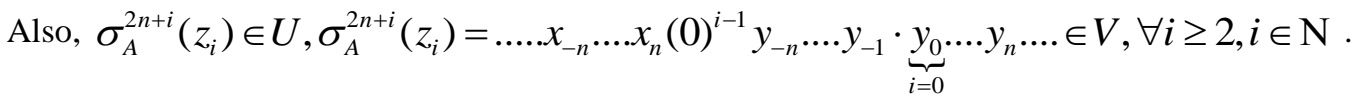

So, $\sigma_{A}^{2 n+i}(U) \bigcap V \neq \phi$, for all $i \geq 2$. Thus $\sigma_{A}^{k}(U) \bigcap V \neq \phi$, for all $k \geq n_{0}=2 n+1$. 
Hence, the shift map $\sigma_{A}: \Sigma_{A} \rightarrow \Sigma_{A}$ is topologically mixing.

Remarks: An alternative but very simple proof of this theorem can be given as an immediate consequence of the proposition 3.1 as follows:

Consider the transition matrix $A=\left[A_{i j}\right]_{m \times m}$ where $A_{i j}=1$ for $i$ or $j=1$ and $A_{i j}=0$ for $i, j>1$. This transition matrix clearly describes the GMLS $m, m(>2) \in \mathrm{N}$. Also, we have,

$$
A=\left[\begin{array}{ccccc}
1 & 1 & 1 & . & 1 \\
1 & 0 & 0 & . & 0 \\
1 & 0 & 0 & . & 0 \\
. & . & . & . & . \\
1 & 0 & 0 & . & 0
\end{array}\right] \quad \text { and } \quad A^{2}=\left[\begin{array}{ccccc}
m & 1 & 1 & . & 1 \\
1 & 1 & 1 & . & 1 \\
1 & 1 & 1 & . & 1 \\
. & . & . & . & . \\
1 & 1 & 1 & . & 1
\end{array}\right]>0
$$

i.e., $A_{i j}^{n}>0$, for $n=2$ and $\forall 1 \leq i, j \leq m$. So, $A$ is aperiodic and hence irreducible. Hence, by Proposition 3.1, $\sigma_{A}: \Sigma_{A} \rightarrow \Sigma_{A}$ is topologically transitive as well as topologically mixing.

Theorem: 3.3: The set $P\left(\sigma_{A}\right)$ of all the periodic points of $\sigma_{A}: \Sigma_{A} \rightarrow \Sigma_{A}$ is dense in $\Sigma_{A}$. Proof: Let $x=\left(x_{i}\right)_{i=-\infty}^{\infty}=\ldots \ldots x_{-k} \ldots \ldots x_{-2} x_{-1} \cdot x_{0} x_{1} \ldots \ldots x_{k} \ldots \ldots \in \Sigma_{A}$ be arbitrary. Now, for any $\varepsilon>0$, however small, we need to show that there exists a periodic point $p \in P\left(\sigma_{A}\right)$ such that $d_{\rho}(x, p)<\varepsilon$. That is, whatever small $\varepsilon>0$ may be, the $\varepsilon$-neighbourhood of $x$ always contains at least one point of $P\left(\sigma_{A}\right)$.

Again, for fixed $\varepsilon>0$ and $\rho>1$, we can always find $n \in \mathrm{N}$ such that $\rho^{-n}<\varepsilon$. Now for the arbitrary point $x=\left(x_{i}\right)_{i=-\infty}^{\infty} \in \Sigma_{A}$, we claim that there always exists a periodic point $p \in P\left(\sigma_{A}\right)$ in the $\varepsilon$-neighbourhood of $x$. We consider the point $p \in \Sigma_{m}$ such that

$$
p=\ldots \ldots . x_{n} 0 x_{-n} \ldots \ldots x_{0} \ldots x_{n} 0 x_{-n} \ldots \ldots x_{-2} x_{-1} \cdot x_{0} x_{1} \ldots \ldots x_{n} 0 x_{-n} \ldots x_{0} \ldots \ldots x_{n} 0 x_{-n} \ldots \ldots \ldots
$$

That is, the point $p$ has been constructed by concatenating the fixed block $W=0 x_{[-n, n]}$ infinitely in both directions. Such a point can always be constructed with the help of any given point. We claim that the point $p$ so constructed is in $\Sigma_{A}$.

We have, $x=\left(x_{i}\right)_{i=-\infty}^{\infty} \in \Sigma_{A} \Rightarrow x_{[-n, n]}=x_{-n} \ldots \ldots x_{-1} x_{0} x_{1} \ldots \ldots \ldots x_{n} \in B\left(\Sigma_{A}\right)$, language of $\Sigma_{A}$ Again, from the definition of GMLSm, evidently 0 can precede and follow any letter of the alphabet $A=\{0,1,2, \ldots, m-1\}$. As a consequence, we have that $p \in \Sigma_{A}$. Further $p \in \Sigma_{A}$ thus constructed is clearly a periodic point of period $2 n+2$ and hence $p \in P\left(\sigma_{A}\right)$.

Also, since $x$ and $p$ both agree in their $(2 n+1)$-central block, so by definition of metric $d_{\rho}$, we have that $d_{\rho}(x, p) \leq \rho^{-n}<\varepsilon$. Thus for any point $x \in \Sigma_{A}$, we have a point $p \in P\left(\sigma_{A}\right)$ which is at a distance less than any given small quantity $\varepsilon>0$. Hence $P\left(\sigma_{A}\right)$ is dense in $\Sigma_{A}$.

Theorem: 3.4: The shift map $\sigma_{A}: \Sigma_{A} \rightarrow \Sigma_{A}$ has sensitive dependence on initial conditions with the sensitivity constant $\delta=1$.

Proof: We show that for any $\varepsilon>0$ and $x=\left(x_{i}\right)_{i=-\infty}^{i=\infty} \in \Sigma_{A}$, there always exists a point $y=\left(y_{i}\right)_{i=-\infty}^{\infty} \in \Sigma_{A}$ in the $\varepsilon$-neighbourhood of $x$ such that $x_{k+1} \neq y_{k+1}$ for some $k \in \mathrm{N}$. Let $\varepsilon>0$ be arbitrary and $N_{\varepsilon}(x)$ be the $\varepsilon$ -neighbourhood of $x$. Then, for fixed $\rho>2 m-1$, there exists a positive integer $n \in \mathrm{N}$ such that $\rho^{-n} \leq \varepsilon<\rho^{1-n}$ and hence clearly we have that

$C_{-n, n}\left(x_{-n}, \ldots, x_{n}\right) \subseteq B_{d_{\rho}}\left(x, \rho^{-n}\right) \subseteq B_{d_{\rho}}(x, \varepsilon)=N_{\varepsilon}(x)$. Let us now choose $y$ such that 


$$
y=\left(y_{i}\right)_{i=-\infty}^{\infty}=x_{[-\infty,-1]} \cdot x_{[0, n]} 0 x_{n+2}^{*} 0 x_{[n+3, \infty]} \text { where } x_{n+2}^{*}=(m-1)-x_{n+2}
$$

Now, $x=\left(x_{i}\right)_{i=-\infty}^{i=\infty} \in \Sigma_{A} \Rightarrow x_{[-\infty,-1]}, x_{[0, n]}, x_{[n+3, \infty]} \in B\left(\Sigma_{A}\right)$, the language of $\Sigma_{A}$

$$
\Rightarrow y=\left(y_{i}\right)_{i=-\infty}^{\infty}=x_{[-\infty,-1]} \cdot x_{[0, n]} 0 x_{n+2}^{*} 0 x_{[n+3, \infty]} \in \Sigma_{A}
$$

This is because of the fact that 0 can precede and follow any letter of the alphabet in all the sequences of a GMLS $m$. Here $x$ and $y$ agree at least in their $(2 n+1)$ central blocks. So clearly we have $d_{\rho}(x, y) \leq \rho^{-n}<\varepsilon$ and

$$
\text { hence } y \in B_{d_{\rho}}\left(x, \rho^{-n}\right)=C_{-n, n}\left(x_{-n}, \ldots, x_{n}\right) \subseteq B_{d_{\rho}}(x, \varepsilon)=N(x) \text {. }
$$

Also,

$$
\begin{gathered}
\sigma^{n+2}(x)=\ldots x_{-n} \ldots x_{0} \ldots x_{n+1} \cdot x_{n+2} \ldots ., \quad \sigma^{n+2}(y)=x_{[-\infty,-1]} x_{[0, n]} 0 \cdot x_{n+2}^{*} 0 x_{[n+3, \infty]}, x_{n+2}^{*} \neq x_{n+2} \\
\Rightarrow \sigma^{n+2}(x) \neq \sigma^{n+2}(y) \text { where }\left(\sigma^{n+2}(x)\right)_{0} \neq\left(\sigma^{n+2}(y)\right)_{0} \\
\Rightarrow d_{\rho}\left(\sigma^{n+2}(x), \sigma^{n+2}(y)\right)=1(=\delta)
\end{gathered}
$$

Thus there exists $\delta(=1)$ such that for any $x=\left(x_{i}\right)_{i=-\infty}^{\infty} \in \Sigma_{A}$ and any neighbourhood $N(x)$ of $x$, there exists $y=\left(y_{i}\right)_{i=-\infty}^{\infty} \in N(x)$ and $k(=n+2) \in \mathrm{N}$ with $d_{\rho}\left(\sigma^{k}(x), \sigma^{k}(y)\right)=1(=\delta)$.

Hence $\sigma_{A}: \Sigma_{A} \rightarrow \Sigma_{A}$ has sensitive dependence on initial conditions.

Theorem: 3.5: The shift map $\sigma_{A}: \Sigma_{A} \rightarrow \Sigma_{A}$ Devaney as well as Auslander-Yorke chaotic.

Proof: We have seen in theorem

(i) 3.2 that $\sigma_{A}$ is topologically transitive

(ii) 3.3 that the set $P\left(\sigma_{A}\right)$ of all the periodic points of $\sigma_{A}$ is dense in $\Sigma_{A}$

(iii) 3.4 that $\sigma_{A}$ has sensitive dependence on initial conditions.

So, it immediately follows that $\sigma_{A}: \Sigma_{A} \rightarrow \Sigma_{A}$ is Devaney chaotic. Also, we know that a Devaney chaotic map is always Auslander-Yorke chaotic. Hence, $\sigma_{A}: \Sigma_{A} \rightarrow \Sigma_{A}$ is also Auslander-Yorke chaotic.

Theorem3.6: The shift map $\sigma_{A}: \Sigma_{A} \rightarrow \Sigma_{A}$ is generically $\delta$-chaotic with $\delta=\operatorname{diam}\left(\Sigma_{A}\right)=1$.

Proof: In the Theorem 3.2, we have proved that the shift transformation $\sigma_{A}: \Sigma_{A} \rightarrow \Sigma_{A}$ is topologically mixing. Also, by proposition 2.2, we know that a continuous topologically mixing map on a compact metric space is topologically weak mixing. So, the shift map $\sigma_{A}$ being a continuous topologically mixing map on the compact metric space $\Sigma_{A}$ is topologically weak mixing.

Again, a continuous topologically weak mixing map on a compact metric space $X$ is generically $\delta$-chaotic on $X$ with $\delta=\operatorname{diam}(X)$, so, it follows that the shift transformation $\sigma_{A}: \Sigma_{A} \rightarrow \Sigma_{A}$ being a continuous topologically weak mixing map on the compact metric space $\Sigma_{A}$ is generically $\delta$-chaotic with

$\delta=\operatorname{diam}\left(\Sigma_{A}\right)=1$.

Theorem: 3.7: The Topological Dynamical System $\left(\Sigma_{A}, \sigma_{A}\right)$ has modified weakly chaotic dependence on initial conditions.

Proof: We first recall that a dynamical system $(X, f)$ has modified weakly chaotic dependence on initial conditions if for any $x \in X$ and for any neighbourhood $N(x)$ of $x$, there are points $y, z \in N(x)$ with $y \neq x, z \neq x$ such that $(y, z) \in X^{2}$ is Li-Yorke.

Let $\rho>2 m-1$ be fixed. Then, for any point $x=\left(x_{i}\right)_{i=-\infty}^{\infty} \in \Sigma_{A}$ let $N(x)$ be any neighbourhood of $x$ in $\Sigma_{A}$. $N(x)$ being a neighbourhood of $x$ in $\Sigma_{A}$ there exists an open set (open nbhd.) $U$ of $\Sigma_{A}$ such that $x \in U \subseteq N(x)$.

Now, since $x \in U$ and $U$ is an open set, so, for some $n \in \mathrm{N}$ we have an open ball $B\left(x, \rho^{-n}\right)$ such that $B\left(x, \rho^{-n}\right) \subseteq U \subseteq N(x)$. Also, since $\rho>2 m-1$, so as a consequence of propositions 2.7 and 2.9 we have 
that $B\left(x, \rho^{-n}\right)$ is the admissible symmetric cylinder $C_{-n, n}\left(x_{-n}, \ldots \ldots, x_{n}\right)$. We now construct two points $y, z \in N(x)$ with $y \neq x, z \neq x$ such that the pair $(y, z) \in \Sigma_{A}^{2}$ is Li-Yorke. We recall that a pair $(y, z) \in \Sigma_{A}^{2}$ is Li-Yorke in $\left(\Sigma_{A}, \sigma_{A}\right)$ with modulus $\delta>0$ if $\lim _{n \rightarrow \infty} \operatorname{Sup}_{\rho}\left(\sigma^{n}(y), \sigma^{n}(z)\right) \geq \delta$ and $\lim \operatorname{Inf} d_{\rho}\left(\sigma^{n}(y), \sigma^{n}(z)\right)=0$. Before proving the theorem, we first define some words $W(x, 2 n)$, $W(x, 6 n), W(x, 10 n)$ etc. of special pattern by using the letters in $x=\left(x_{i}\right)_{-\infty}^{\infty} \in \Sigma_{A}$ for the simplification of our proof as follows:

$$
\begin{aligned}
& W(x, 2 n)=0 x_{2 n+2}^{*} 0 x_{2 n+4}^{*} 0 \ldots \ldots . .0 x_{4 n}^{*} 0 x_{4 n+2} \ldots \ldots \ldots x_{6 n}, \\
& W(x, 6 n)=0 x_{6 n+2}^{*} 0 x_{6 n+4}^{*} 0 \ldots \ldots . .0 x_{8 n}^{*} 0 x_{8 n+2} \ldots \ldots \ldots x_{10 n} \\
& W(x, 10 n)=0 x_{10 n+2}^{*} 0 x_{10 n+4}^{*} 0 \ldots \ldots \ldots .0 x_{12 n}^{*} 0 x_{12 n+2}^{*} \ldots \ldots \ldots x_{14 n}, \ldots . . \text { and so on. }
\end{aligned}
$$

Note that all the above words are of the type $W(x, 2(2 k-1) n), k \in \mathrm{N}$, and are constructed in such a way that every word contains $4 n$ letters starting at the place $2(2 k-1) n+1$ for the word $W(x, 2(2 k-1) n)$. Also, in every word all the letters in the odd places of the first $2 n$ places are 0 's and those of even places are $m$-nary complements of the corresponding letters in $x$, the letter in $(2 n+1)$-th place is 0 and all the letters in the rest $(2 n-1)$ places are just the letters in the corresponding places of $x$. Further in all the above words $x_{k}^{*}=(m-1)-x_{k}, \forall k$, the $m$-nary complement of $x_{k}$.

Now with the help of the above words we construct the points $y, z \in \Sigma_{A}$ as follows:

$$
\begin{aligned}
& y=\ldots \ldots x_{-n} \ldots \ldots x_{-1} \cdot \underbrace{x_{0}}_{i=0} x_{1} \ldots \ldots x_{n} 0 x_{n+2}^{*} 0 x_{n+4}^{*} 0 \ldots \ldots .0 x_{2 n}^{*} 0 x_{2 n+2} \ldots \ldots x_{6 n} x_{6 n+1} x_{6 n+2} \ldots \ldots \ldots \ldots . . . \text { and } \\
& z=\ldots \ldots x_{-n} \ldots x_{-1} \cdot \underbrace{x_{0} \ldots \ldots}_{i=0} x_{n} 0 x_{n+2}^{*} 0 \ldots \ldots 0 x_{2 n}^{*} W(x, 2 n) W(x, 6 n) W(x, 10 n) W(x, 14 n) \ldots \ldots \ldots . . . \text { Here }
\end{aligned}
$$

$x=\left(x_{i}\right)_{i=-\infty}^{\infty} \in \Sigma_{A} \Rightarrow x_{[-\infty, n]} \in B\left(\Sigma_{A}\right)$, the language of $\Sigma_{A}$. Also, since in a GMLSm, 0 can precede and follow any letter of the corresponding alphabet, so, all the words $W(x, 2 n), W(x, 6 n), W(x, 10 n)$ etc. are allowed blocks in $\Sigma_{A}$ and consequently $y, z \in \Sigma_{A}$. We note here that $z$ contains infinitely many words of the type $W(x, 2(2 k-1) n)$, where $k \in \mathrm{N}$, containing $4 n$ letters each.

With the above notations in mind we now prove the theorem as follows: $y, z$ agree with $x$ in the $(2 n+1)$-central block

$$
\Rightarrow d_{\rho}(x, y)<\rho^{-n}, d_{\rho}(x, z)<\rho^{-n} \text { and hence } y, z \in B\left(x, \rho^{-n}\right) \subseteq U \subseteq N(x)
$$

Also,

$$
\begin{aligned}
& \sigma^{2 n+2}(y)=x_{[-\infty, n]} 0 x_{n+2}^{*} 0 \ldots 0 x_{2 n}^{*} 0 \cdot \underbrace{x_{2 n+2}}_{i=0} x_{2 n+3} \ldots \ldots . x_{3 n} \ldots \ldots x_{4 n} \ldots \ldots . . x_{5 n} \ldots \ldots \ldots x_{6 n} x_{6 n+1} \ldots \ldots \ldots . \\
& \sigma^{2 n+2}(z)=x_{[-\infty, n]} 0 x_{n+2}^{*} 0 \ldots 0 x_{2 n}^{*} 0 \cdot \underbrace{x_{2 n+2}^{*}}_{i=0} 0 \ldots 0 x_{6 n}^{*} W(x, 6 n) W(x, 10 n) W(x, 14 n) \ldots
\end{aligned}
$$

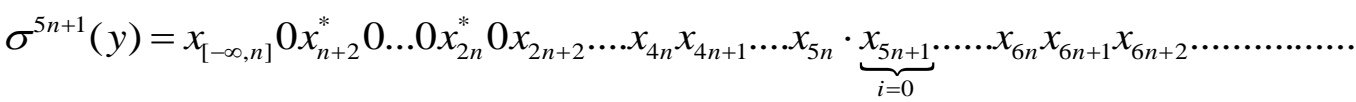

$$
\begin{aligned}
& \sigma^{5 n+1}(z)=z_{[-\infty, 4 n]} 0 x_{4 n+2} x_{4 n+3} \ldots x_{5 n} \cdot \underbrace{x_{5 n+1}}_{i=0} x_{5 n+2} \ldots x_{6 n} W(x, 6 n) W(x, 10 n) W(x, 14 n) \ldots \ldots . .
\end{aligned}
$$

Here $\left(\sigma^{2 n+2}(y)\right)_{0} \neq\left(\sigma^{2 n+2}(z)\right)_{0}$ and $\sigma^{5 n+1}(y), \sigma^{5 n+1}(z)$ agree at least in $(2 n-1)$-central block. So, from these we immediately have that

$$
\begin{aligned}
& \text { (i) } d_{\rho}\left(\sigma^{2 n+2}(y), \sigma^{2 n+2}(z)\right)=1 \text { and } \\
& \text { (ii) } d_{\rho}\left(\sigma^{5 n+1}(y), \sigma^{5 n+1}(z)\right) \leq \rho^{-(n-1)}
\end{aligned}
$$

Therefore, 


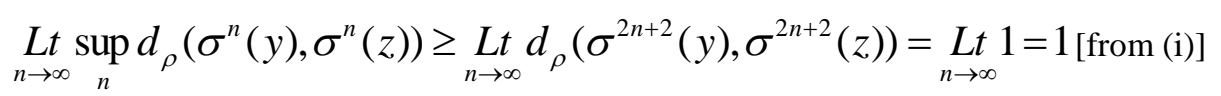

Again, $0 \leq \operatorname{Lt}_{n \rightarrow \infty} \inf _{n} d_{\rho}\left(\sigma^{n}(y), \sigma^{n}(z)\right) \leq \operatorname{Lt}_{n \rightarrow \infty} d_{\rho}\left(\sigma^{5 n+1}(y), \sigma^{5 n+1}(z)\right) \leq \underset{n \rightarrow \infty}{L t} \rho^{-(n-1)}=0$ [from (ii)]

Now, $0 \leq \operatorname{Lt}_{n \rightarrow \infty} \inf _{n} d_{\rho}\left(\sigma^{n}(y), \sigma^{n}(z)\right) \leq 0 \Rightarrow \operatorname{Lt}_{n \rightarrow \infty} \inf _{n} d_{\rho}\left(\sigma^{n}(y), \sigma^{n}(z)\right)=0$.

Thus $\underset{n \rightarrow \infty}{\operatorname{Lt}} \sup _{n} d_{\rho}\left(\sigma^{n}(y), \sigma^{n}(z)\right) \geq 1 \quad$ and $\underset{n \rightarrow \infty}{\operatorname{Linf}} \inf _{\rho} d_{\rho}\left(\sigma^{n}(y), \sigma^{n}(z)\right)=0$.

So, $(y, z) \in \Sigma_{A}^{2}$ is a Li-Yorke pair with modulus $\delta=1>0$. Hence, the dynamical system $\left(\Sigma_{A}, \sigma_{A}\right)$ has modified weakly chaotic dependence on initial conditions.

\section{Theorem: 3.8: The dynamical system $\left(\Sigma_{A}, \sigma_{A}\right)$ has chaotic dependence on initial conditions.}

Proof: We know that a dynamical system $(X, f)$ has chaotic dependence on initial conditions if for any $x \in X$ and every neighbourhood $N(x)$ of $x$, there exists a $y \in N(x)$ such that the pair $(x, y) \in X^{2}$ is $L i$ Yorke.

Let, $a=\left(a_{i}\right)_{i=-\infty}^{\infty} \in \Sigma_{A}$ be arbitrary and $N(a)$ be any neighbourhood of $a$. Then there exists an open set $U$ in $\Sigma_{A}$ such that $a \in U \subseteq N(a)$. Now, since $a \in U$ and $U$ is an open set in $\Sigma_{A}$, so for some $n \in \mathrm{N}$, there exists an open ball $B_{d_{\rho}}\left(a, \rho^{-n}\right)$ such that $B_{d_{\rho}}\left(a, \rho^{-n}\right) \subseteq U \subseteq N(a)$.

Fix $\rho>2 m-1$ so that $B_{d_{\rho}}\left(a, \rho^{-n}\right)=C_{-n, n}\left(x_{-n}, \ldots, x_{n}\right)$. Now with the help of the letters in $a$, we construct a point $b \in B_{d_{\rho}}\left(a, \rho^{-n}\right) \subseteq U \subseteq N(a)$ such that $(a, b) \in \Sigma_{A}^{2}$ is Li-Yorke.

Using the letters in $a=\ldots \ldots . . a_{-3} a_{-2} a_{-1} \cdot a_{0} a_{1} a_{2} \ldots \ldots . a_{n} \ldots \ldots \in \Sigma_{A}$, we define the words $W(a, 2 n), W(a, 6 n)$, $W(a, 10 n), \ldots$ etc. as follows:

$$
\begin{aligned}
& W(a, 2 n)=0 a_{2 n+2}^{*} 0 a_{2 n+4}^{*} 0 \ldots \ldots .0 a_{4 n}^{*} \mathrm{O} a_{4 n+2} \ldots \ldots \ldots a_{6 n}, \\
& W(a, 6 n)=0 a_{6 n+2}^{*} \mathrm{O} a_{6 n+4}^{*} 0 \ldots \ldots . .0 a_{8 n}^{*} 0 a_{8 n+2} \ldots \ldots \ldots a_{10 n}, \\
& W(a, 10 n)=0 a_{10 n+2}^{*} 0 a_{10 n+4}^{*} 0 \ldots \ldots . .0 a_{12 n}^{*} 0 a_{12 n+2} \ldots \ldots . . . a_{14 n}, \ldots . . \text { and so on. }
\end{aligned}
$$

Note that all the above words are of the type $W(a, 2(2 k-1) n), k \in \mathrm{N}$, and are constructed in such a way that every word contains $4 n$ letters starting at the place $2(2 k-1) n+1$ for the word $W(a, 2(2 k-1) n)$. Also, in every word all the letters in the odd places of the first $2 n$ places are 0 's and those of even places are $m$-nary complements of the corresponding letters in $a,(2 n+1)$-th letter is 0 and all the letters in the rest $(2 n-1)$ places are just the letters in the corresponding places of $a$. Further $a_{k}^{*}=(m-1)-a_{k}, \forall k$, the $m$-nary complement of $a_{k}$.

Now we take

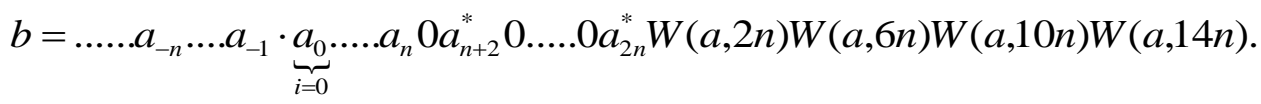

$a=\left(a_{i}\right)_{i=-\infty}^{\infty} \in \Sigma_{A} \Rightarrow a_{[-\infty, n]} \in B\left(\Sigma_{A}\right)$, the language of $\Sigma_{A}$. Also, in every sequence of a GMLSm, 0 can precede and follow any letter of the corresponding alphabet. So, the word $0 a_{n+2}^{*} 0 \ldots 0 a_{2 n}^{*}$ and all the words $W(a, 2 n), W(a, 6 n), W(a, 10 n)$ etc. are allowed blocks in $\Sigma_{A}$ and consequently $b \in \Sigma_{A}$.

From the construction of $b$ it is clear that $b$ agrees with $a$ in $(2 n+1)$-central block. So, we get, $d_{\rho}(a, b)<\rho^{-n}$ and hence $b \in B_{d_{\rho}}\left(a, \rho^{-n}\right) \subseteq U \subseteq N(a)$.

Also, $\quad b^{\prime}=\sigma^{2 n+2}(b)=a_{[-\infty, n]} 0 a_{n+2}^{*} 0 \ldots 0 a_{2 n}^{*} 0 \cdot \underbrace{a_{2 n+2}^{*}}_{i=0} 0 \ldots 0 a_{4 n}^{*} 0 a_{4 n+2} \ldots a_{6 n} W(a, 6 n) W(a, 10 n) \ldots \ldots .$. And $\sigma^{5 n+1}(b)=b_{[-\infty, 4 n]}^{\prime} 0 a_{4 n+2} \ldots \ldots . a_{5 n} \cdot \underbrace{a_{5 n+1}}_{i=0} a_{5 n+2} \ldots \ldots . a_{6 n} W(a, 6 n) W(a, 10 n) W(a, 14 n) \ldots \ldots \ldots . .$. Here, $\quad$ we 
see that $\left(\sigma^{2 n+2}(a)\right)_{0} \neq\left(\sigma^{2 n+2}(b)\right)_{0}$ and $\sigma^{5 n+1}(a), \sigma^{5 n+1}(b)$ agree at least in $(2 n-1)$-central block. So, from these observations we immediately have that

$$
\begin{aligned}
& \text { (A) } d_{\rho}\left(\sigma^{2 n+2}(a), \sigma^{2 n+2}(b)\right)=1 \text { and (B) } \underset{n \rightarrow \infty}{L t} d_{\rho}\left(\sigma^{5 n+1}(a), \sigma^{5 n+1}(b)\right) \leq \rho^{-(n-1)} \text { Therefore, } \\
& \underset{n \rightarrow \infty}{L t} \sup _{n} d_{\rho}\left(\sigma^{n}(a), \sigma^{n}(b)\right) \geq \underset{n \rightarrow \infty}{L t} d_{\rho}\left(\sigma^{2 n+2}(a), \sigma^{2 n+2}(b)\right)=\underset{n \rightarrow \infty}{L t} 1=1 \quad \text { [from (A)] }
\end{aligned}
$$

Again, $0 \leq \operatorname{Lt}_{n \rightarrow \infty} \inf _{n} d_{\rho}\left(\sigma^{n}(a), \sigma^{n}(b)\right) \leq \operatorname{Lt}_{n \rightarrow \infty} d_{\rho}\left(\sigma^{5 n+1}(a), \sigma^{5 n+1}(b)\right) \leq \underset{n \rightarrow \infty}{L t} \rho^{-(n-1)}=0$ [from (B)]

Now, $0 \leq \underset{n \rightarrow \infty}{L t} \inf _{n} d_{\rho}\left(\sigma^{n}(a), \sigma^{n}(b)\right) \leq 0 \Rightarrow \underset{n \rightarrow \infty}{L t} \inf _{n} d_{\rho}\left(\sigma^{n}(a), \sigma^{n}(b)\right)=0$.

Thus

$\underset{n \rightarrow \infty}{L t} \sup _{n} d_{\rho}\left(\sigma^{n}(a), \sigma^{n}(b)\right) \geq 1 \quad$ and $\quad \underset{n \rightarrow \infty}{L t} \inf _{n} d_{\rho}\left(\sigma^{n}(a), \sigma^{n}(b)\right)=0$.

Hence, $(a, b) \in \Sigma_{m}^{2}$ is a Li-Yorke pair with modulus $\delta=1>0$. Consequently, the dynamical system $\left(\Sigma_{A}, \sigma_{A}\right)$ has chaotic dependence on initial conditions.

\section{Zeta functions for maps}

Consider a dynamical system $(X, f)$. For $n \in \mathrm{N}$, let $p_{n}(f)$ denotes the number of periodic points of period $n$ of the map $f$ i.e., $p_{n}(f)=\left|\left\{x \in X: f^{n}(x)=x\right\}\right|$.Then $p_{n}$ is a topological invariant [1]. The zeta function [1,4] $\zeta_{f}(t)$ of $f$, is again a topological invariant [1] which combines all the $p_{n}^{\text {'s }}$. For a dynamical system $(X, f)$ with $p_{n}(f)<\infty, \forall n \in \mathrm{N}$, the zeta function $\zeta_{f}(t)$ is defined as follows:

$$
\zeta_{f}(t)=\exp \left(\sum_{n=1}^{\infty} \frac{p_{n}(f)}{n} t^{n}\right)
$$

Expanding out the powers of the series gives,

$$
\zeta_{f}(t)=1+p_{1}(f) t+\frac{1}{2}\left[p_{2}(f)+p_{1}(f)^{2}\right] t^{2}+\frac{1}{6}\left[2 p_{3}(f)+3 p_{2}(f) p_{1}(f)+p_{1}(f)^{3}\right] t^{3}+
$$

For example, consider the dynamical system $\left(\Sigma_{A}, \sigma_{A}\right)$ where $\Sigma_{A}$ is the Golden Mean shift such that it is described by the transition matrix $A=\left[\begin{array}{ll}1 & 1 \\ 1 & 0\end{array}\right]$. Then, if $\lambda=\frac{1+\sqrt{5}}{2}$ and $\mu=\frac{1-\sqrt{5}}{2}$ be the eigen values of the transition matrix $A=\left[\begin{array}{ll}1 & 1 \\ 1 & 0\end{array}\right]$, then,

$$
\begin{aligned}
& p_{n}\left(\sigma_{A}\right)=\operatorname{tr}\left(A^{n}\right)=\lambda^{n}+\mu^{n} \\
& \therefore \quad \zeta_{\sigma_{A}}(t)=\exp \left(\sum_{n=1}^{\infty} \frac{p_{n}\left(\sigma_{A}\right)}{n} t^{n}\right)=\exp \left(\sum_{n=1}^{\infty} \frac{\lambda^{n}+\mu^{n}}{n} t^{n}\right) \\
&= \exp \left(\sum_{n=1}^{\infty} \frac{(\lambda t)^{n}}{n}+\sum_{n=1}^{\infty} \frac{(\mu t)^{n}}{n}\right) \\
&= \exp (-\log (1-\lambda t)-\log (1-\mu t)) \\
&= \frac{1}{(1-\lambda t)(1-\mu t)}=\frac{1}{1-t-t^{2}}
\end{aligned}
$$

The most important technique to derive the zeta function of the shift map of any shift of finite type is given in the following theorem:

Theorem: 4.1[1]: If A be a $r \times r$ non-negative integer matrix, $\chi_{A}(t)$ be its characteristic polynomial and $\sigma_{A}$ its associated shift map, then 


$$
\zeta_{\sigma_{A}}(t)=\frac{1}{t^{r} \chi_{A}\left(t^{-1}\right)}=\frac{1}{\left|\mathrm{I}_{r}-t A\right|}=\frac{1}{\prod_{\lambda \in s p^{\mathrm{X}}(A)}(1-\lambda t)}, \text { where } \operatorname{sp}^{\mathrm{X}}(A) \text { is the nonzero spectrum of } A .
$$

4.2: Derivation of zeta function for the shift map $\sigma_{A}$ on the GMLSm: We know that GMLS $m$ is described by the non-negative integer matrix $A$ given by:

$$
A=\left[\begin{array}{ccccc}
1 & 1 & 1 & \cdot & 1 \\
1 & 0 & 0 & . & 0 \\
1 & 0 & 0 & . & 0 \\
\cdot & \cdot & \cdot & \cdot & \cdot \\
1 & 0 & 0 & . & 0
\end{array}\right]_{m \times m}=\left[\begin{array}{cc}
A_{11} & B \\
C & O
\end{array}\right] \text {, where } A_{11}=[1], B=\left[\begin{array}{c}
1 \\
1 \\
\cdot \\
\cdot \\
1
\end{array}\right]_{(m-1) \times 1}^{T}, C=\left[\begin{array}{c}
1 \\
1 \\
. \\
1
\end{array}\right]_{(m-1) \times 1},
$$

and $O$ is the zero matrix of order $(m-1) \times(m-1)$

Here to find the zeta function of the shift map $\sigma_{A}$ on GMLSm, by fruitfully using the theorem 4.1, we need to compute $\left|I_{m}-t A\right|$. We perform this as follows:

$$
\begin{aligned}
D=\left|\mathrm{I}_{m}-t A\right| & =\left|\begin{array}{cccccc}
1-t & -t & -t & -t & . . & -t \\
-t & 1 & 0 & 0 & . . & 0 \\
-t & 0 & 1 & 0 & . . & 0 \\
-t & 0 & 0 & 1 & . . & 0 \\
. . & . . & . . & . . & . . & . . \\
-t & 0 & 0 & 0 & . . & 1
\end{array}\right|_{m \times m} \\
& =\sum_{j=1}^{m} d_{1 j} \cdot C_{1 j} \text { where } C_{1 j} \text { is the cofactor of element } d_{1 j} \text { in } D \\
& =(1-t)\left|\mathrm{I}_{m-1}\right|-t \cdot C_{12}-t \cdot C_{13}-t \cdot C_{14}-\cdots-t \cdot C_{1 m} \\
& =(1-t) \cdot 1+t^{2} \cdot\left|\mathrm{P}_{12}\right|-t^{2} \cdot\left|\mathrm{P}_{13}\right|+t^{2} \cdot\left|\mathrm{P}_{14}\right|-\cdots+(-1)^{m} t \cdot\left|\mathrm{P}_{1 m}\right|
\end{aligned}
$$

Here $\mathrm{P}_{1 j}, 2 \leq j \leq m, j \in \mathrm{N}$, is the permutation matrix obtained from $\mathrm{I}_{m-1}=\left(e_{1}, e_{2}, \ldots, e_{m-1}\right)$ by switching $e_{1}$ to $(j-1)^{t h}$ row. Then, $\left|\mathrm{P}_{1 j}\right|=(-1)^{j-1}$ and consequently we get,

$$
\begin{gathered}
D=\left|\mathrm{I}_{m}-t A\right|=(1-t) \cdot 1+t^{2} \cdot\left|\mathrm{P}_{12}\right|-t^{2} \cdot\left|\mathrm{P}_{13}\right|+t^{2} \cdot\left|\mathrm{P}_{14}\right|-\cdots+(-1)^{m} t^{2} \cdot\left|\mathrm{P}_{1 m}\right| \\
=(1-t)-\underbrace{\left[t^{2}+t^{2}+t^{2}+t^{2}+t^{2}+\ldots \ldots+t^{2}\right]}_{(m-1) \text { summands }} \\
=1-t-(m-1) t^{2} \\
\therefore \zeta_{\sigma_{A}}(t)=\frac{1}{t^{r} \chi_{A}\left(t^{-1}\right)}=\frac{1}{\left|\mathrm{I}_{m}-t A\right|}=\frac{1}{1-t-(m-1) t^{2}} \ldots \ldots \ldots \ldots \ldots \ldots \ldots \ldots \ldots \ldots \ldots \ldots \ldots \ldots
\end{gathered}
$$

In case of Golden Mean shift we have found that $\zeta_{\sigma_{A}}(t)=\frac{1}{1-t-t^{2}}$

This may be obtained by putting $\mathrm{m}=2$ in [1].

\section{Conclusions}

In this paper we have mainly established that the shift map on the Golden Mean Lookalike shift of order $m$ [GMLS $m$ ] is Devaney Chaotic. To do this we have employed the concepts of graphs, linear algebra, topological Markov chains and metric spaces. In theorem 3.4, the well-known chaotic shift transformation $\sigma_{A}$ on $\Sigma_{A}$ have been shown to be generically $\delta$-chaotic with $\delta=\operatorname{diam}\left(\Sigma_{A}\right)=1$. In theorem 3.6 and 3.7, we have 
proved that $\sigma_{A}$ has respectively modified weakly chaotic dependence and weakly chaotic dependence on initial conditions. In the proofs of both the theorems, Li-Yorke pairs have been constructed in a very clear-cut way and the concepts of cylinders and admissible cylinders have been extensively used. Further, we have derived the zeta function of this transformation. The methods of establishing some results may be fruitfully employed for the same purpose in other topological Markov chains. Most of the results are quite interesting and might have profound applications in analysis and in discrete mathematics.

\section{References}

[1]. Lind, Douglas, and Marcus, Brian, Introduction to Symbolic Dynamics and Coding, Cambridge University Press, Cambridge, New York, 1995

[2]. Katok, Anatole and Hasselblatt, Boris, Introduction to the Modern Theory of Dynamical Systems, Cambridge University Press, New York, 1999

[3]. Alligood, Kathleen T. et al, CHAOS-An Introduction to Dynamical Systems, Springer-Verlag New York, Inc., 175 Fifth Avenue, New York, 2008

[4]. Brin, Michael and Stuck, Garret, Introduction to Dynamical Systems, Cambridge University Press, New York, USA, 2002.

[5]. Kitchens, B.P., Symbolic Dynamics: One-sided, Two-sided and Countable State Markov Shifts, Universitext, Springer Verlag, Berlin, 1998.

[6]. Devaney, R.L., An Introduction to Chaotic Dynamical Systems, $2^{\text {nd }}$ Edition, Addison-Wesly, Redwood City, CA, 1989.

[7]. Zuoling, Zhou, The Topological Markov Chain, Acta Mathematica Sinica, New Series, 1988, Vol.4 No.4, pp. 330-337.

[8]. Mangang, K. Binod, Topological Entropy of Golden Mean Lookalike Shift spaces, International Journal of Mathematics and Statistics Invention, Volume 2 Issue 7, pp.05-10, July 2014.

[9]. Banks, J.et al., On Devaney's Definition of Chaos, The American Mathematical Monthly,vol.99 No.4, pp.332-334, 1992

[10]. Dutta, T. K. and Burhagohain, A., Some Properties of the Chaotic Shift Map on the Generalised m-symbol Space and Topological Conjugacy, International Journal of Mathematics Research, Vol.7 No.2, 2015, pp. 173-190.

[11]. Bhaumik, I and Choudhury, B. Y., The Shift Map and the Symbolic Dynamics and Application of Topological Conjugacy, Journal of Physical Sciences, Vol.13, 2009, pp. 149-160.

[12]. Blanchard, F. et al., On Li-Yorke Pairs, J. Reine Angew. Math., 547, pp. 51-68, 2002.

[13]. Dutta ,T. K. and Burhagohain, A., Chaotic Aspects of the Shift Map on the Bi-sided Full m-Shift, International Journal of Applied Mathematics and Statistical Sciences, Vol.5, Issue 4, 2016, pp. 71-91.

[14]. Adams, Colin and Franzosa, Robert, Introduction to Topology: Pure and Applied, First Edition, Pearson Education, Inc, 2008. 